\title{
Mediastinal small cell cancer associated with Lambert-Eaton myasthenic syndrome: A case report
}

\author{
KEJIAN ZHANG, WEI LIU, YANG LI, KEWEI ZHANG, XINLIANG GAO and JING WANG \\ Department of General Thoracic Surgery, Bethune Chest Center, Bethune First Hospital of Jilin University, \\ Changchun, Jilin 130021, P.R. China
}

Received June 27, 2014; Accepted February 16, 2015

DOI: $10.3892 / \mathrm{etm} .2015 .2454$

\begin{abstract}
Lambert-Eaton myasthenic syndrome (LEMS) is typically characterized as a paraneoplastic syndrome associated with small cell lung carcinoma. The presence of LEMS in association with other neuroendocrine lung tumors, including carcinoids or large cell lung carcinoma, is highly unusual. The present case study reports the very rare case of a patient with mediastinal small cell cancer combined with LEMS. A 53-year-old man was admitted to the Bethune First Hospital with a 3-month history of weakness in the lower extremities that had become aggravated during the previous 20 days. A positron emission tomography-computed tomography (CT) examination demonstrated multiple enlarged, hypermetabolic lymph nodes between the vena cava and trachea, indicating the presence of inflammation. No other abnormalities were identified. A mediastinal lymph node biopsy revealed a high prevalence of small cell neuroendocrine carcinoma. The immunohistochemistry results were as follows: Ki-67 (+50, meaning that $50 \%$ of the cells exhibited Ki-67 expression), cytokeratin $(\mathrm{CK} ;+)$, cluster of differentiation (CD)56 (+), synaptophysin $(+)$, thyroid transcriptional factor-1 (+), epithelial membrane antigen $(+)$, neuron-specific enolase $(+)$, P63 (weakly $+)$, leukocyte common antigen (-), CD99 (-), vimentin (-) and CK5/6 (-). Following a diagnosis of LEMS, the patient underwent one course of cisplatin + etoposide chemotherapy, which
\end{abstract}

Correspondence to: Dr Wei Liu, Department of General Thoracic Surgery, Bethune Chest Center, Bethune First Hospital of Jilin University, 71 Xinmin Street, Changchun, Jilin 130021, P.R. China E-mail: davidliuw135@163.com

Abbreviations: LEMS, Lambert-Eaton myasthenic syndrome; T-LEMS, tumorous Lambert-Eaton myasthenic syndrome; NT-LEMS, non-tumorous Lambert-Eaton myasthenic syndrome; SCLC, small cell lung carcinoma; EMG, electromyography; RNS, repetitive nerve stimulation; CMAP, compound muscle action potential; $\mathrm{CT}$, computed tomography; PET-CT, positron emission tomography-computed tomography; CK, cytokeratin; $\mathrm{CD}$, cluster of differentiation; VGCC, voltage-gated calcium channel

Key words: small cell lung cancer, Lambert-Eaton myasthenic syndrome, mediastinal was ineffective. Subsequently, the patient underwent a different chemotherapy regimen (cisplatin + etoposide + ifosfamide), which significantly improved the symptoms of myasthenia. Following completion of this treatment regimen, the primary lesion was not observed on a lung CT scan, and the mediastinal lymph nodes had clearly diminished. Subsequently, the patient underwent two courses of radiotherapy (40 Gy/22f/6W).

\section{Introduction}

One of the most commonly affected sites in a number of diseases is the neuromuscular synapse. The neuromuscular junction is involved in various conditions, such as autoimmune diseases, genetic polymorphisms and toxic disorders. The most prominent symptom in all such conditions is muscular weakness, which is caused by the interference of acetylcholine-mediated transmission of signals from the presynaptic nerve to the skeletal muscle, which subsequently impairs muscle contraction (1). Autoimmune, genetic and toxic conditions are known to affect this transmission either presynaptically or postsynaptically (2).

Lambert-Eaton myasthenic syndrome (LEMS) is one such autoimmune disease. This syndrome, also termed myasthenic syndrome, affects acetylcholine release at the presynaptic membrane of the neuromuscular junction (3). LEMS can be divided into tumorous LEMS (T-LEMS) and non-tumorous LEMS (NT-LEMS) forms (4). The condition is typically recognized as a paraneoplastic syndrome associated with small cell lung carcinoma (SCLC); the association of LEMS with other neuroendocrine lung tumors, including carcinoids or large cell lung carcinoma, is highly unusual (5). T-LEMS is more often observed in a clinical setting compared with NT-LEMS, and SCLC is the most commonly associated type of tumor in patients with T-LEMS. However, the clinical observation of mediastinal small cell cancer in association with LEMS is extremely rare. A number of patients are commonly misdiagnosed, resulting in delayed treatment (6). The aim of the present case report was to provide further understanding with regard to the diagnosis and treatment of T-LEMS. Thus, we herein describe a rare case of LEMS in association with an atypical mediastinal carcinoid tumor, which exhibited transient clinical and electrophysiological remission following surgical resection, chemotherapy and radiotherapy (7). 


\section{Case report}

A 53-year-old man was admitted to the Bethune First Hospital (Changchun, China) with a 3-month history of weakness in the lower extremities that had become aggravated during the previous 20 days. Three months earlier, the patient had begun to feel weakness in the lower limbs, extending from the inguinal region downward to the knees, without a known muscle weakness. The patient reported aggravation of this weakness during the previous 20 days, most notably a lack of strength in the two lower limbs, although the patient was able to walk unaided. Weakness of the upper limbs was also experienced, although the patient was able to hold things independently. The patient also reported the symptom of a dry mouth. No breathing difficulties or drooping eyelids were observed. The patient had a $>20$-year smoking history involving an average of 20 cigarettes per day, as well as a $>20$-year history of occasional alcohol use.

A physical examination revealed no abnormalities of consciousness, language fluency, memory, orientation or calculation ability. Bilateral pupil dilation was present, and the pupils were round, of the same size, and sensitive to light. Both eyeballs moved freely without nystagmus. The bilateral nasolabial grooves were deep, and the patient's tongue was oriented along the midline when protruded. The muscle tension in the limbs was normal. More specifically, the muscle strength in the upper limbs was normal, and the muscle strength in the lower limbs was graded as $4+$, indicating that the patient was able to resist gravity and perform a resistance movement with $75 \%$ of normal muscle strength. The patient exhibited no marked ataxia, and the pathological reflex was negative for the lower limbs. No evident abnormalities were identified during the whole-body examination.

An auxiliary examination revealed no abnormalities in the thyroid function test, serum electrolyte levels or tumor marker levels. In addition, no marked findings were observed with the electrocardiography or magnetic resonance imaging of the head, cervical vertebrae and thoracic vertebrae. The neostigmine test result was negative. With regard to the electromyography (EMG), low-frequency repetitive nerve stimulation (RNS) resulted in a $>15 \%$ reduction in the compound muscle action potential (CMAP) amplitude, and high-frequency RNS resulted in a $>100 \%$ increase in the CMAP amplitude. The EMG examination demonstrated a typical presynaptic membrane neuromuscular transmission obstacle, which was in accordance with LEMS. A chest computed tomography (CT) scan revealed no abnormalities. However, positron emission tomography (PET)-CT (Fig. 1A and B) revealed enlargement and hypermetabolic activity of multiple lymph nodes between the vena cava and trachea, which indicated the presence of inflammation. No other abnormalities were identified from the scans. Therefore, the patient was diagnosed with LEMS. Following the hospitalization period, a mediastinal lymph node biopsy under thoracoscopic guidance was performed due to the absence of histological pathology. The pathology report indicated a high prevalence of small cell neuroendocrine carcinoma (Fig. 2A). Hematoxylin and eosin staining revealed oat cells and short spindle cells, nuclear hyperchromatism, and indistinct nucleoli. The immunohistochemistry results were as follows: Cluster of differentiation (CD)56 (+; Fig. 2B), cytokeratin (CK; +; Fig. 2C), Ki-67 (+50, meaning that 50\% of the cells exhibited Ki-67 expression; Fig. 2D), synaptophysin (+; Fig. 2E), thyroid transcriptional factor-1 (+; Fig. 2F), epithelial membrane antigen $(+)$, neuron-specific enolase $(+)$, P63 (weakly + ), leukocyte common antigen (-), CD99 (-), vimentin (-) and CK5/6 (-). Immunohistological figures for epithelial membrane antigen, neuron-specific enolase, P63, leukocyte common antigen, CD99, vimentin and CK5/6 not shown due to poor image quality.

Based on the aforementioned observations, the patient was clinically diagnosed with LEMS accompanied with mediastinal small cell carcinoma. The patient underwent one course of chemotherapy, consisting of $80 \mathrm{mg} / \mathrm{m}^{2}$ cisplatin on day 1 and $100 \mathrm{mg} / \mathrm{m}^{2}$ etoposide on days $1-3$; however, the patient failed to respond. Subsequently, the patient underwent a different chemotherapy regimen, which significantly improved the symptoms. This regimen consisted of $80 \mathrm{mg} / \mathrm{m}^{2}$ cisplatin on day $1,100 \mathrm{mg} / \mathrm{m}^{2}$ etoposide on days $1-3$ and $1.2 \mathrm{~g} / \mathrm{m}^{2}$ ifosfamide on days 1-3. This second regimen was administered four times, with each course lasting four days and a 21-day interval between each course. Written informed consent was obtained from the patient. The primary lesion was not identified on the lung CT images following the treatment. Additionally, the mediastinal lymph nodes were clearly diminished (Fig. 1C and D). Subsequently, the patient underwent two courses of radiotherapy (40 Gy/22f/6W).

\section{Discussion}

LEMS is an autoimmune disease involving the dysregulation of acetylcholine release at the presynaptic membrane of the neuromuscular junction (3). The symptoms of the condition resemble those of myasthenia gravis. LEMS is often associated with tumors, and 50-60\% of patients with LEMS have an underlying tumor (T-LEMS). SCLC has been observed in $90 \%$ of T-LEMS cases. By contrast, NT-LEMS is often associated with the human leukocyte antigen-B8-DR3 haplotype and a variety of other autoimmune diseases, such as rheumatoid arthritis, pernicious anemia, thyroid disorders and Sjögren's syndrome (7).

In the majority of cases, LEMS involves muscle fatigue and weakness of the limbs and trunk. Lower limb weakness is more common compared with upper limb weakness; similarly, the proximal aspects of the limbs are more commonly affected than the distal aspects. Dominant cranial nerve muscles are not generally involved. A small number of patients have symptoms of drooping eyelids, double vision, difficulty swallowing and dysarthria. Muscle weakness is felt in the resting state, and strength increases after a few seconds due to the vigorous contracting of muscles. Evidence suggests that half of patients with LEMS also exhibit disruption of the autonomic nervous system. These individuals may experience a dry mouth, impotence and impaired tear secretion and sweating (8). Furthermore, in a small number of patients, an impaired performance of certain regions of the central nervous system, such as the cerebellum, can be observed (4).

In the majority of patients with LEMS, muscle weakness appears prior to symptoms of cancer. There have only been a few cases where tumors have been diagnosed earlier than LEMS (9). Therefore, careful screening to detect any possible 

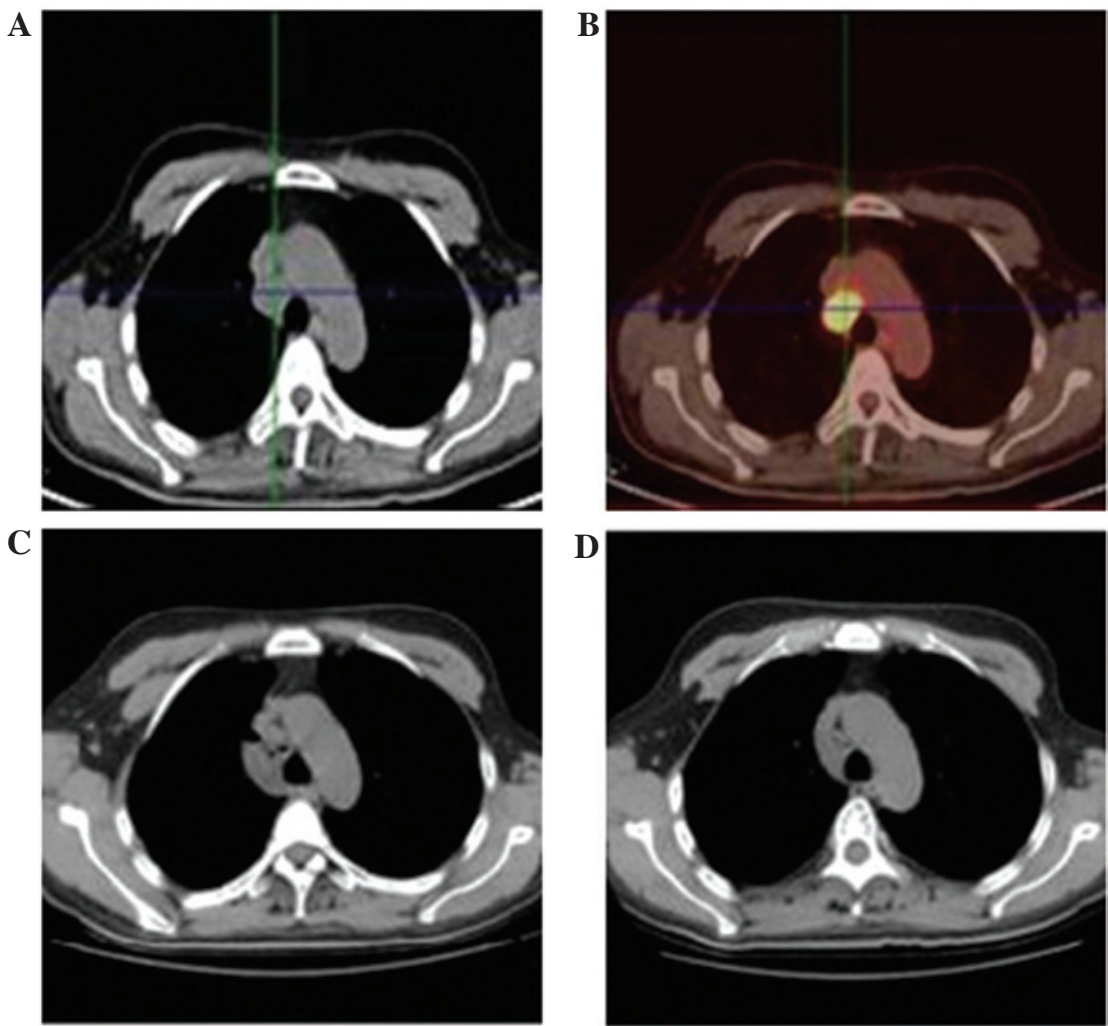

Figure 1. Computed tomography (CT) lung scans of a 53-year-old patient who was hospitalized with symptoms of muscle weakness and a suspicion of Lambert-Eaton myasthenic syndrome. CT was performed at different time points during the clinical course: Presurgical (A) chest computed and (B) positron emission tomography-CT scans. (C) Postsurgical, prechemotherapeutic and (D) following two courses of chemotherapy (the mediastinal lymph nodes were clearly diminished).
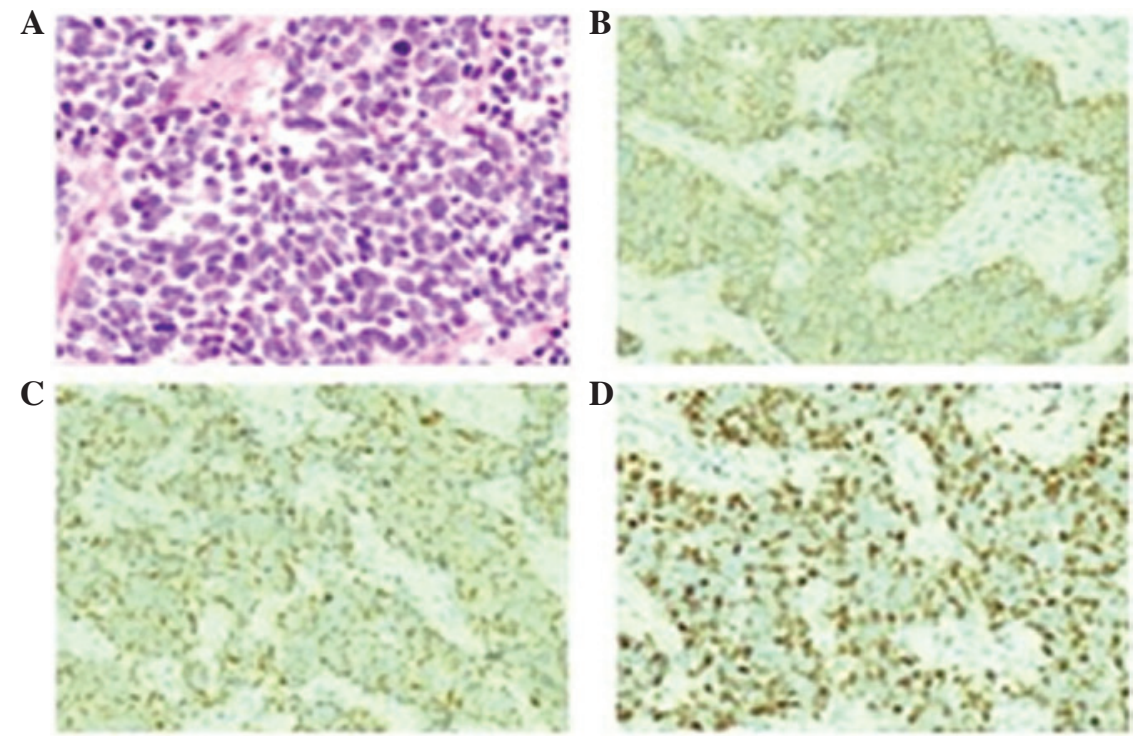

$\mathbf{E}$

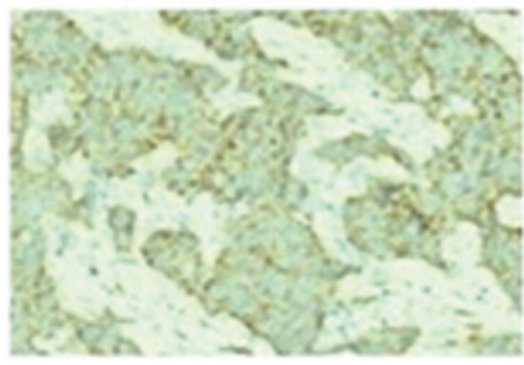

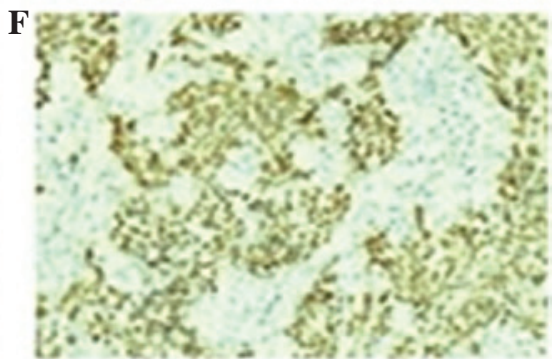

Figure 2. Histological and immunohistochemical findings of the mediastinal small cell tumor. (A) Hematoxylin and eosin staining revealed oat cells and short spindle cells, nuclear hyperchromatism, and indistinct nucleoli. (B-F) Immunohistochemistry staining of paraffin-embedded sections demonstrated (B) cluster of differentiation $56(+),(C)$ cytokeratin (+), (D) Ki-67 (+50), (E) synaptophysin (+) and (F) thyroid transcriptional factor-1 (+) cells (magnification, x400). 
associated cancers at an early stage is a crucial step in optimal disease management (7).

A timely diagnosis of LEMS is crucial not only to ensure proper treatment of the neurological disease, but also to detect any underlying tumors early in the course of the disease. However, early diagnosis is often hampered by the rarity of LEMS and its fluctuating symptoms (4). Even when the patient's symptoms have been properly associated with a neuromuscular transmission disorder, LEMS is often misdiagnosed as myasthenia gravis. Clinical suspicion of LEMS should be confirmed with an EMG. Although weakness is more evident in the proximal limbs, electrophysiological abnormalities are more easily detected in the distal muscles. Therefore, the diagnosis of LEMS is based on the presence of the typical triad, comprising a low CMAP amplitude at rest $(0.1-6.0 \mathrm{mV})$, a further decrease in the CMAP amplitude during low-frequency RNS $(2-5 \mathrm{~Hz})$, and an increase in the CMAP amplitude during high-frequency RNS $(20-50 \mathrm{~Hz})$ or immediately after a brief maximal voluntary contraction (15-20 sec). The latter (an increase in the CMAP amplitude during high-frequency RNS or immediately after a brief maximal voluntary contraction) is the technique of choice due to the higher level of tolerance (7). $A \geq 100 \%$ increase in the CMAP amplitude/area (post-tetanic/postexercise facilitation) in at least two tested muscles is fairly typical of LEMS, with a sensitivity ranging between 84 and 96\% (4). Low-frequency RNS during maximal contraction may increase the sensitivity of the EMG. In addition, single-fiber EMG may be more sensitive compared with RNS; however, the technique does not distinguish between myasthenia gravis and LEMS (5). In the present case, the EMG results revealed that the CMAP amplitude with low-frequency RNS diminished by $>15 \%$, while that with high-frequency RNS increased by $>100 \%$.

Antibodies targeting the P/Q-type voltage-gated calcium channel (VGCC) are present in $75-90 \%$ of patients with LEMS. In patients with T-LEMS, this rate can further increase to $100 \%$, and the condition is generally associated with high antibody titers (4). Research has indicated that monitoring the anti-P/Q-type VGCC serum antibody titer may be important for evaluating the efficacy of chemotherapy for LEMS associated with SCLC (10). Based on the two aforementioned positive findings, the patient in the current study was definitively diagnosed with LEMS with mediastinal small cell cancer.

Treatment of T-LEMS should initially target the malignant tumor. Thus, surgical resection should be performed to remove the primary tumor, followed by chemotherapy and radiation therapy. The symptoms of LEMS, including muscle weakness, have been reported to improve following this treatment regimen (4).

In patients with T-LEMS, treatment of the associated tumor generally induces a significant improvement in the neurological symptoms. Severely affected patients requiring a rapid therapeutic response may benefit from high-dose prednisone in combination with plasmapheresis or intravenous immunoglobulin (11). However, the use of immunosuppressants other than steroids in patients with T-LEMS is controversial; the general trend is to avoid such agents as much as possible, although there is no definite contraindication to azathioprine treatment (7).

In the present case report, the patient underwent a chemotherapy regimen described in an earlier study (4). Briefly, $80 \mathrm{mg} / \mathrm{m}^{2}$ cisplatin was administered on day 1 and $100 \mathrm{mg} / \mathrm{m}^{2}$ etoposide was administered on days 1-3. However, the patient failed to respond. Subsequently, the patient underwent a different chemotherapy regimen, consisting of $80 \mathrm{mg} / \mathrm{m}^{2}$ cisplatin on day $1,100 \mathrm{mg} / \mathrm{m}^{2}$ etoposide on days $1-3$ and $1.2 \mathrm{~g} / \mathrm{m}^{2}$ ifosfamide on days 1-3, which significantly improved the symptoms of myasthenia. Furthermore, the mediastinal lymph nodes were found to have clearly diminished. The patient completed four courses of chemotherapy and two courses of radiotherapy (40 Gy/22f/6W). Following treatment, the muscle strength returned to normal, and the patient was able to freely move his limbs during normal activities. The patient is continuing to undergo follow-up.

The present case report serves as a reminder that in patients with T-LEMS, muscle weakness occurs prior to the presentation of tumor-related symptoms. Once a patient is diagnosed with LEMS, the selection of the most appropriate treatment is dependent upon the correct identification of the underlying cause, which may be a tumor or an additional autoimmune disease.

In conclusion, routine examinations, including chest $\mathrm{CT}$, PET-CT, fiber bronchoscopy and pathological examination, can lead to a definitive diagnosis of T-LEMS. In cases of successful post-treatment tumor regression, the patient should undergo regular follow-up examinations over a rational period of time to ensure the early detection of tumor recurrence.

\section{Acknowledgements}

This study was supported by a grant from the National Natural Science Foundation of China (no. 81272607).

\section{References}

1. Lee JH, Shin JH, Kim DS, et al: A case of Lambert-Eaton myasthenic syndrome associated with atypical bronchopulmonary carcinoid tumor. J Korean Med Sci 19: 753-755, 2004.

2. Gilhus NE: Myasthenia and the neuromuscular junction. Curr Opin Neurol 25: 523-529, 2012.

3. Tomiyasu K, Ito H, Kanazawa N, Saito T and Kowa H: Anti-Hu antibody in a patient with Lambert-Eaton myasthenic syndrome and early detection of small cell lung cancer. Intern Med 34: 1082-1085, 1995.

4. LoMonaco M, Milone M, Padua L and Tonali P: Combined low-rate nerve stimulation and maximal voluntary contraction in the detection of compound muscle action potential facilitation in Lambert-Eaton myasthenic syndrome. Muscle Nerve 20: 1207-1208, 1997.

5. Titulaer MJ, Lang B and Verschuuren JJ: Lambert-Eaton myasthenic syndrome: from clinical characteristics to therapeutic strategies. Lancet Neurol 10: 1098-1107, 2011.

6. Martín-García E, Mannara F, Gutiérrez-Cuesta J, et al: Intrathecal injection of $\mathrm{P} / \mathrm{Q}$ type voltage-gated calcium channel antibodies from paraneoplastic cerebellar degeneration cause ataxia in mice. J Neuroimmunol 261: 53-59, 2013.

7. Wu X, Wang J, Liu Y and Liu K: Clinical presentation and differential diagnosis of Lambert-Eaton myasthenic syndrome. Neurosciences (Riyadh) 18: 169-172, 2013.

8. Nakamur S, Kawagishi Y, Kato S, et al: Long-term survival case of small-cell lung cancer with Lambert-Eaton myasthenic syndrome without anticancer therapy. Nihon Kokyuki Gakkai Zasshi 48: 918-922, 2010 (In Japanese).

9. Baggi F, Ubiali F, Nava S, et al: Effect of IgG immunoadsorption on serum cytokines in MG and LEMS patients. J Neuroimmunol 201-202: 104-110, 2008.

10. Tsukiji J, Kaneko T, Saito H, et al: A case of cT0N2M0 small cell lung cancer with Lambert-Eaton myasthenic syndrome. Nihon Kokyuki Gakkai Zasshi 42: 820-824, 2004 (In Japanese).

11. Oh SJ, Kurokawa K, Claussen GC and Ryan HF Jr: Electrophysiological diagnostic criteria of Lambert-Eaton myasthenic syndrome. Muscle Nerve 32: 515-520, 2005. 